\title{
Lymphatic imaging to assess rheumatoid flare: mechanistic insights and biomarker potential
}

\author{
Homaira Rahimi ${ }^{1,2,9^{*}}$ (D), Richard Bell ${ }^{1,3}$, Echoe M. Bouta ${ }^{1,4}$, Ronald W. Wood ${ }^{5,6,8}$, Lianping Xing ${ }^{1,3}$, \\ Christopher T. Ritchlin ${ }^{1,7}$ and Edward M. Schwarz ${ }^{1,3,4,6,7}$
}

\begin{abstract}
Proliferation of draining lymphatic vessels coupled with dynamic changes in lymph node volume and flow are characteristic features in rheumatoid arthritis (RA). Furthermore, impaired lymph egress from inflamed synovium is associated with joint flare in murine models of inflammatory-erosive arthritis. Unfortunately, advances towards a greater understanding of lymphatic changes in RA pathogenesis have been slow due to the absence of outcome measures to quantify lymphatic function in vivo. While lymphoscintigraphy is the current standard to assess lymphedema and sentinel lymph nodes in cancer patients, its sensitivity and specificity are inadequate to study lymphatics in RA. The emergence of high-resolution MRI, power Doppler ultrasound, and near-infrared imaging that permits real-time quantification of lymphatic function in animal models has been a major advance, and these techniques have produced a new paradigm of altered lymphatic function that underlies both acute arthritic flare and chronic inflammation. In acute flare, lymphatic drainage increases several fold, whereas no lymphatic contractions are detected in lymph vessels draining chronic arthritic joints. Moreover, these outcomes are now being adapted to study lymphatics in RA towards the development of novel biomarkers of arthritic flare and the discovery of new therapeutic targets. In particular, interventions that directly increase lymphatic egress from diseased joints by opening collateral lymphatic vessels, and that restore lymphatic vessel contractions, provide novel therapeutic approaches with potential for minimal toxicity and immunosuppression. To summarize the origins of this field, recent advances, and future directions, we herein review: current knowledge of lymphatics in RA based on classic literature; new in-vivo imaging modalities that have elucidated how lymphatics modulate acute versus chronic joint inflammation in murine models; and how these preclinical outcome measures are being translated to study lymphatic function in RA inflammation and how effective RA therapies alter lymphatic flow and lymph nodes draining flaring joints.

Trial registration: ClinicalTrials.gov NCT02680067. Registered 7 December 2015; ClinicalTrials.gov NCT01098201. Registered 30 March 2010; and ClinicalTrials.gov NCT01083563. Registered 8 March 2010.

Abbreviations: ${ }^{99} \mathrm{Tc}$, Technetium99; B-in cells, B-cells-in-inflamed-nodes; CE, Contrast enhanced; CZP, Certolizumab; DMARD, Disease-modifying anti-rheumatic drug; LN, Lymph node; MCP, Metacarpophalangeal; NIR, Near-infrared imaging; ICG, Indocyanine green; PD, Power Doppler; US, Ultrasound; PIP, Proximal interphalangeal; RA, Rheumatoid arthritis; TNF-Tg, Tumor necrosis factor transgenic; tt, Transit time
\end{abstract}

\footnotetext{
* Correspondence: Homaira_Rahimi@urmc.rochester.edu

${ }^{1}$ Center for Musculoskeletal Research, University of Rochester School of

Medicine and Dentistry, Rochester, NY, USA

${ }^{2}$ Department of Pediatrics, University of Rochester School of Medicine and

Dentistry, Rochester, NY, USA

Full list of author information is available at the end of the article
} 


\section{Background}

Rheumatoid arthritis (RA) is a chronic, debilitating disease affecting over 1 million people in the United States, and $1 \%$ of the population worldwide [1]. In the current etiological paradigm, autoimmunity to citrullinated peptides drives a systemic inflammatory response that is expressed most strongly in the joints, although a range of other tissues can be affected. However, this model does not explain the events that drive episodic occurrences, known clinically as arthritic or rheumatic flares, and remissions, a hallmark of this disease. RA can also be viewed as a multifactorial syndrome that arises from an interaction of predetermined and stochastic factors which foster disease onset and persistence [2]. This perspective highlights the major unmet clinical burden shouldered by up to $30 \%$ of RA patients refractory to current therapies, and by the larger population that suffers from sudden exacerbation of joint pain which may be accompanied by aggressive cartilage and bone catabolism. Although ubiquitously used in both clinical and preclinical studies [3-5], characterizing the term "flare" has been challenging; however, current efforts are underway to create a uniform set of parameters to define RA flare [6]. What is widely accepted is that flare can occur in newly diagnosed RA patients or in patients with longstanding, chronic arthritis. It is important to note that these enigmatic flares often appear in the setting of long-term effective therapy, sometimes without detectable changes in systemic inflammatory status as measured by inflammatory markers. To better understand whether local factors can trigger arthritic flare, investigators turned their attention to lymphatic drainage, and demonstrated that changes in lymphatic vessel structure and function may be instrumental in provoking joint inflammation $[7,8]$. These discoveries were facilitated by novel imaging methods that provided quantitative assessment of lymphatic vessel function for the first time. These advances provide new opportunities to understand how lymphatic biomarkers may be applied to aid in diagnosis of arthritic flare and to assess therapeutic effectiveness. The mechanistic pathways that maintain lymph flow provide new therapeutic targets which may restore dysfunctional lymphatics. In this review, we will: outline the current knowledge of lymphatic function as it relates to RA; describe how contemporary lymphatic imaging methods reveal a central role for lymphatics in arthritic flare; and point out the diagnostic and therapeutic potential of emerging lymphatic vessel biomarkers in RA.

\section{Lymphatic vasculature structure and function}

It is important to understand normal lymphatic vasculature anatomy in order to appreciate when lymphatic dysfunction occurs. A primary purpose of the lymphatic vasculature is to preserve fluid homeostasis within an organism [9]. Lymphatic vessels allow for reabsorption of extravascular and interstitial fluid via blind-ended lymphatic capillaries that become more organized collecting lymphatic vessels. These vessels can drain to lymph nodes (LNs) and are termed afferent vessels. Collecting vessels that drain lymph from a $\mathrm{LN}$ are termed efferent vessels. In humans, most of the vessels and LNs in this network ultimately reach the thoracic duct, which collects the lymph and drains it into the blood circulatory system via the left subclavian vein. A small portion of lymph fluid is collected by the right lymphatic duct, which reaches the blood circulation via the right subclavian vein [10]. Most mammals, including mice and rats, have a similar anatomy - that is, thoracic duct drainage of lymph into the blood vasculature [11] - and thus are useful preclinical disease models. Lymph movement results from multiple mechanisms including intrinsic contraction of collecting vessels, as well as the presence of valves in collecting vessels that prevent retrograde movement and thus promote forward movement of lymph. Lymph movement also occurs via an intrinsic contraction associated with movement of lymph as boluses; the mechanisms underlying and regulating this contractile activity remain unclear and are an area of intense study.

\section{Mechanisms of lymphangiogenesis in inflammation}

Research did not focus on the development and growth of lymphatic vessels until the mid-1990s. Initial discoveries by Alitalo and colleagues found vascular endothelial growth factor receptor 3 (VEGFR-3) as a marker for lymphatic vessels in mouse and human tissue, and lent credence to the theory of a venous origin for lymphatic tissue; shortly thereafter, further research from the group identified VEGF-C as the specific ligand for VEGFR-3 $[12,13]$. Work from our group further delineated the mechanism of lymphangiogenesis in inflammatory arthritis [14-16]. In the tumor necrosis factor-transgenic (TNF-Tg) mouse, we found increased expression of VEGF-C and increased lymphatic vessel formation in the synovium of these mice early on during the disease process. At 2.5 months old, when clinical parameters such as paw swelling are beginning, the TNF-Tg mice were treated with VEGFR-3 blocking antibody to inhibit VEGF-C binding. After 8 weeks of treatment, we noted decreased lymphatic vessel formation and popliteal lymph node (PLN) size, and increased evidence of joint inflammation as determined by synovial volumes and synovial inflammatory areas at the ankle and knee joints. These findings led us to conclude that VEGF-C-induced lymphangiogenesis ameliorates joint tissue damage in arthritis by promoting drainage of inflamed joints. Furthermore, our in-vitro studies showed that TNF 
stimulates osteoclast precursors to express VEGF-C and promote lymphangiogenesis. However, persistent inflammation may inhibit lymphatic vessel development and draining function. Recently, we found that TNF stimulates lymphatic endothelial cells to produce iNOS, which inhibits lymphatic smooth muscle cell contraction and lymph drainage [17]. An early study by Polzer et al. [18] reported that specific blockade of TNF was associated with increased lymphangiogenesis in a TNF-Tg mouse model and in RA and spondyloarthritis synovial tissues, suggesting a parallel mechanism in human disease [18]. They theorized that formation of more lymphatic vessels would improve clearance of pathogenic mediators, including proinflammatory cytokines and effector immune cells, such as macrophages. It should be noted that they used a different model of the TNF-Tg mouse, which develops severe inflammatory disease earlier than our model. The finding that both TNF and TNF inhibition can stimulate lymphangiogenesis, although counterintuitive, may therefore relate to the location, stage, and extent of inflammation. It is clear, however, that further research will be necessary to clarify the role of TNF in lymphangiogenesis and inflammation.

\section{Lymphatics in RA}

Although Chauffard and Ramond first described LN involvement and lymphadenopathy in RA patients in 1896 $[19,20]$, remarkably little is still known about the pathologic features and underling etiology. Harold Paulus and colleagues proposed that effector lymphocytes in lymphatic fluid were central to RA pathogenesis. In support of this view was the dramatic improvement of joint symptoms in RA patients who underwent drainage of the thoracic duct [21]. They described a cohort of nine women with untreated RA aged 24-65 years old who underwent insertion of a fistula into the thoracic duct and drainage of lymphatic fluid. Cells were removed from the fluid and reinfused; the depletion was performed daily for 19-105 days. Within 1 week, the investigators documented improvement in the number of tender and swollen joints, decreased morning stiffness, and improved grip strength. Unfortunately, symptoms returned after removal of the fistula and completion of the study. In subsequent experiments they infused autologous labeled lymphocytes, and arthritic flares were noted a mean of 3.5 days after administration. These findings supported their hypothesis that lymphocytes in the lymphatic fluid are the primary mediators of RA [22-24]. Despite the new knowledge gained in these studies, further advances using this approach were stalled due to the lack of visual and quantitative assessments coupled with the challenges related to the feasibility of the procedure and transient responses. Indeed, the entire literature on lymphatic outcomes in arthritis is limited to three peer-reviewed publications from 1937 to 2001 [25-27].

\section{Classical lymphoscintigraphy imaging of arthritis in the clinic}

Historically, performance of lymphoscintigraphy in arthritis patients was limited to those with lymphedema. In 1968, Kallioma and Vastamak [28] reported on lymphoscintigraphy studies in Finland of the lymphatics in two women with RA who subsequently developed lymphedema of the right arm. They noted decreased uptake in the axillary nodes of the lymphedematous arthritic hand at both timepoints, and proposed an association between lymphatic dysfunction and inflammatory arthritis. Subsequently, radiocolloid lymphoscintigraphy became the imaging modality of choice over the lymphogram, an invasive procedure that requires cannulation and injection of lymphatic vessels with contrast in order to visualize them with X-ray imaging [29].

In the 1990s, several studies used ${ }^{99}$ technetium $\left({ }^{99} \mathrm{Tc}\right)$ colloid lymphoscintigraphy to demonstrate lymphatic dysfunction in patients with RA or psoriatic arthritis who developed lymphedema in their arthritic extremities [30-33]. In the majority of these case reports, lymphedema lessened when arthritis improved after therapy; this was associated with normalization of colloid uptake assessed by lymphoscintigraphy. However, improvement was not associated with the duration of lymphedema, arthritis, or the type of disease-modifying anti-rheumatic drug (DMARD). The co-occurrence of RA and lymphedema is a relatively rare event so opportunities to gain additional insights are limited; gaining more understanding of the role of lymphatics in RA requires an alternative strategy.

In our initial human studies we turned to lymphoscintigraphy to analyze lymphatic transport in RA inflammation pre and post anti-TNF therapy as part of a clinical study (ClinicalTrials.gov NCT01083563). The duration of treatment was 18 weeks, and patients were scanned just prior to initiation of therapy and after 18 weeks. An example of these ${ }^{99} \mathrm{Tc}$ sulfur colloid tracing studies is shown in Fig. 1. Colloid was injected in the usual fashion into the web spaces of the fingers or toes by a radiologist, and the limb was scanned at specific timepoints. Because lymphoscintigraphy cannot identify individual lymphatic vessels, it cannot detect lymphatic contractions. The technique therefore relies on the transit time (tt) of colloid from the injection site to $\mathrm{LN}$ regions, and compares the tt with known normal times. This study produced interesting findings, but we abandoned this approach due to the relatively invasive nature of the technique, radiation exposure, and the prolonged duration of the procedure; these considerations limit its use in the long-term, large-scale studies necessary to further understand the role of lymphatic flow during flare. 

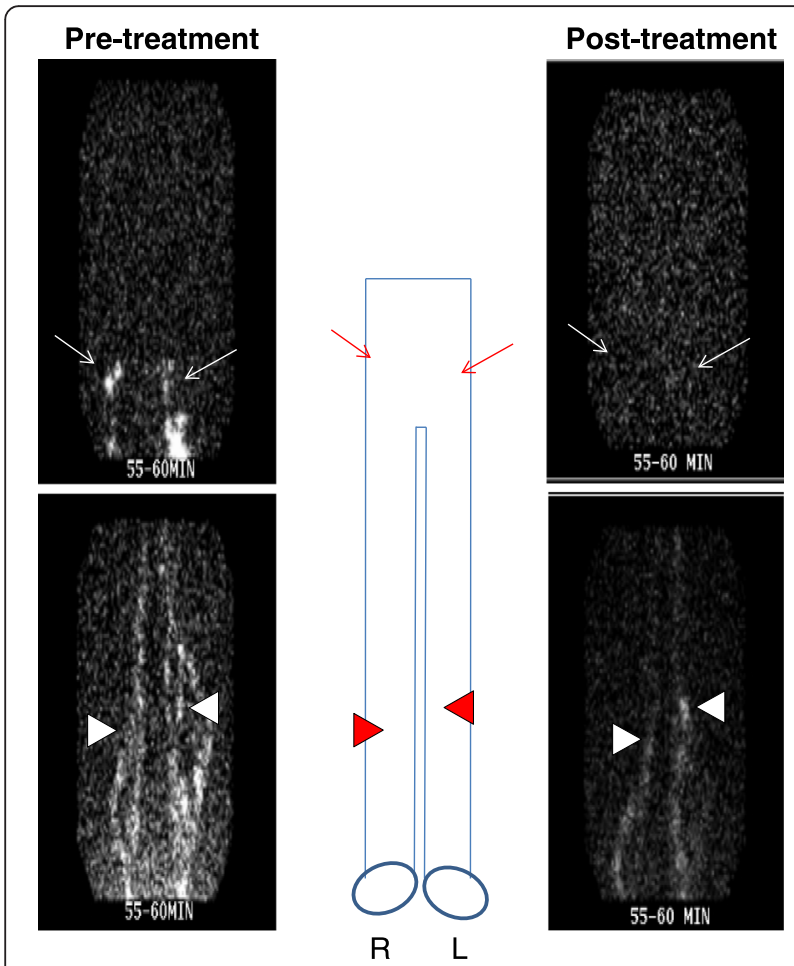

Fig. 1 Increased lymphatic flow from flaring joints normalizes with effective anti-TNF therapy. Images of ${ }^{99} \mathrm{TC}$ sulfur colloid tracing of the lower extremity to the pelvis obtained from a RA patient with asymmetric knee flare enrolled in a clinical study to assess the effects of anti-TNF therapy on arthritis and lymphatics (ClinicalTrials.gov NCT01083563). Note the bright ${ }^{99} \mathrm{TC}$ sulfur colloid signal in the inguinal (arrows) and popliteal (arrowheads) areas on both lower extremities pre treatment, and the decreased signal post treatment, which resulted in normalization of the tt from 15 minutes to 60 minutes. Schematic diagram between the lymposcintigrams orients the regions of interest. $R$ right leg, $L$ left leg

\section{Contrast-enhanced MRI of draining LNs $M R I$ in animal models}

To formally address the barriers of real-time in-vivo imaging of lymphatics in RA patients, we turned to contrastenhanced (CE) MRI to assess the natural history of inflammatory-arthritis in the TNF-Tg and $\mathrm{K} / \mathrm{BxN}$ murine models of inflammatory-erosive arthritis [34-36]. Although MRI methods to assess synovial inflammation in arthritis were well established [37], visualization of lymphatics was never documented. Subsequent CE-MRI studies in TNF-Tg (3647 line) mice revealed that the draining LN undergoes dynamic transitions which are associated with activity of the arthritis in the proximal joint [38-40]. In the initial "expanding" phase, lymphatic fluid flows at an increased rate and volume from an affected joint to the draining LN via afferent lymphatic vessels. The LN volume expands, presumably to accommodate the influx of cells and lymph exiting the inflamed synovium. Furthermore, a subset of B cells, termed B-cells-in-inflamednodes (B-in cells), accumulates in follicular areas of the draining LN [41, 42]. Following an extended expanding phase (typically from 3 to 6 months of age in this model), a stochastic event leads to asymmetric "collapse" of the LN and lymphatic vessels in series along the ipsilateral axis $[42,43]$. Coincident with the collapse, B-in cells translocate to the paracortical sinuses of the $\mathrm{LN}$, the proximal joint swells, and joint histopathology shows synovitis. Additionally, intravital immunofluorescent microscopy studies pre and post treatment with anti-CD20 antibodies that ameliorated arthritic inflammation in TNF-Tg mice demonstrated that translocated B-in cells effectively block passive lymph flow through the LN. Further support of the important function of the B-in cells was the restoration of lymphatic flow by B-cell depletion therapy [38]. Interestingly, while B-cell depletion allowed for restoration of passive flow and amelioration of synovial inflammation, it did not restore the lymphatic contractions or active lymphatic transport. Thus, B cells play a passive role in an arthritic flare that lessens in severity when passive lymphatic egress from inflamed joints occurs. This finding suggested two novel intervention strategies currently under investigation: treatment with agents that restore lymphatic contraction and active lymphatic flow; and nonimmunosuppressive treatments that open collateral lymphatic vessels to restore local lymphatic egress by circumventing the sites of B-cell obstruction. A third concept arising from these studies focused on the involvement of lymphatic smooth muscle cells in dysfunction of lymphatics in RA inflammation. Molecular mechanisms responsible for impaired lymphatic smooth muscle cells and their progenitors/stem cells in RA are another active research focus.

\section{MRI in clinical studies}

To test whether RA pathogenesis also involves expanding and collapsed draining LNs, we completed a clinical pilot study in which patients with knee flare were imaged with CE-MRI pre and post certolizumab (CZP) therapy (ClinicalTrials.gov NCT01098201). Consistent with the natural history of inflammatory-erosive arthritis in murine models, 2D CE-MRI of patients with newly diagnosed RA who had a flare was associated with highly vascular knee synovitis, bone marrow changes, and very large "expanded" PLN (Fig. 2a). The 3D rendering for volumetric analyses confirmed this finding (Fig. 2b). In contrast, 2D MRI of patients with chronic arthritis (disease duration $>20$ years) who developed a flare revealed end-stage synovitis adjacent to focal bone erosions and tiny PLN (Fig. 2c). Volumetric analysis confirmed the predicted "moth-eaten" appearance of the synovium, where vascularity and perfusion is compromised by interdigitating necrotic pannus tissue, and "collapsed" PLN (Fig. 2d). Quantitatively, the total LN volume in 7 of 10 patients with measurable LN on CE-MRI decreased 16 weeks after CZP therapy (mean decrease 

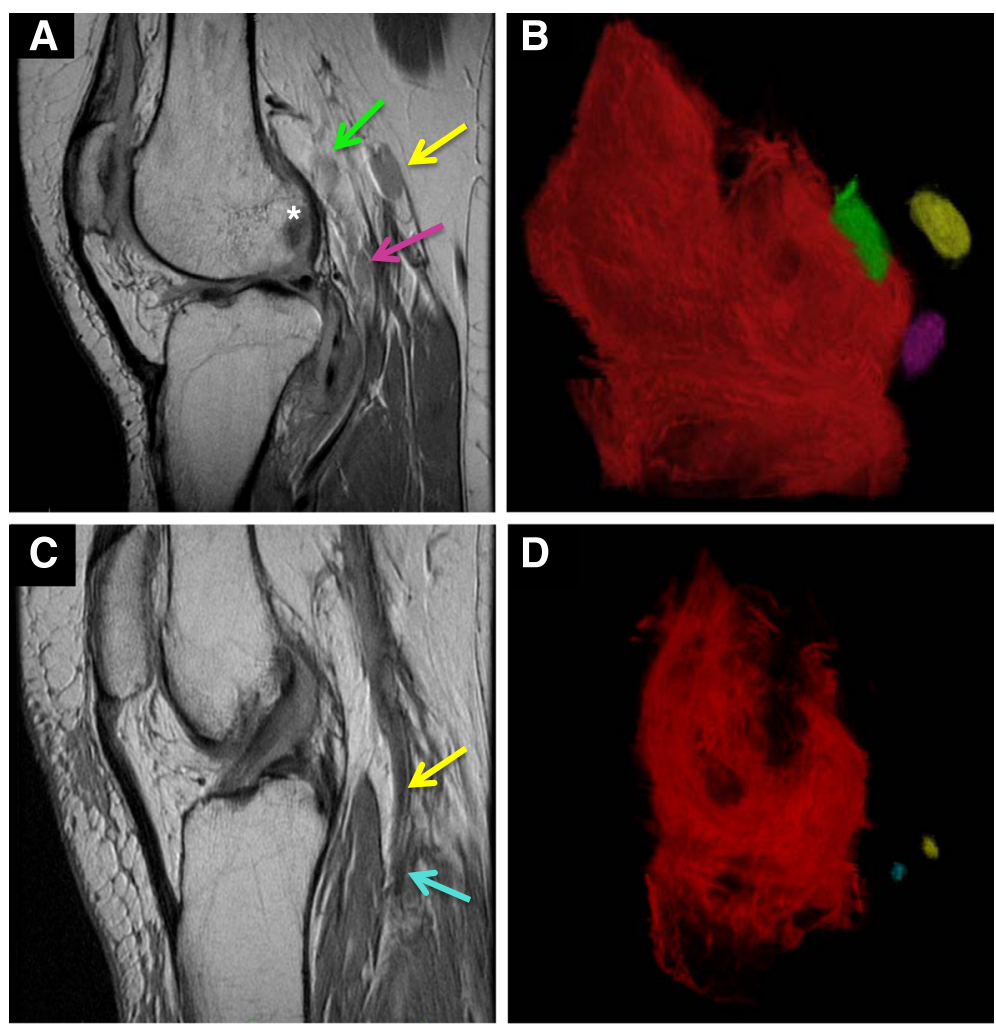

Fig. 2 Expanding versus collapsed popliteal LNs in early versus late-stage RA knee flare. MRIs were obtained from RA patients with new-onset $(\mathbf{a}, \mathbf{b})$ and long-standing (>20 years) $(\mathbf{c}, \mathbf{d})$ asymmetric knee flare, who were enrolled in a phase 4 clinical trial to assess the effects of anti-TNF therapy on PLN volume (ClinicalTrials.gov NCT01098201). The 2D sagittal views $(\mathbf{a}, \mathbf{c})$ and 3D volume renderings $(\mathbf{b}, \mathbf{d})$ illustrate the dramatic differences between early RA flare with expanded PLN (pink, green and yellow arrows corresponding to pseudocolored PLN) adjacent to highly inflamed synovium and focal erosions in the lateral femoral condyle $\left({ }^{*}\right)$, compared with the barely visible collapsed PLN (blue and yellow arrows corresponding to pseudocolored PLN) adjacent to advanced pannus tissue and extensive bone erosions

$37 \% ; p<0.002)$. Significant improvement in knee pain measured by the Rheumatoid and Arthritis Outcome Score for the knee [44] inversely correlated with decrease in PLN volume $\left(R^{2}=0.94\right)$. Collectively, these data indicate that RA patients who sustain draining LN volume during anti-TNF therapy experienced the greatest pain relief in flaring joints, and provide the first clinical evidence to support the concept that dynamic changes in LN volume correlate with response to therapy in RA.

\section{MRI of lymphatics as biomarkers}

Based on the aforementioned preclinical and clinical studies, there is reasonable evidence to support the use of MRI in evaluating draining LNs as a potential early marker of arthritic flare. While the murine models of arthritis have more fully defined that collapse of a draining $\mathrm{LN}$ is associated with arthritic flare, a similar correlation in humans has yet to be elucidated. We know, for example, that LNs undergo dramatic changes during synovial inflammation [45], and that these changes can differentiate between RA versus osteoarthritis. However, MRI alone cannot currently evaluate lymphatic vessels, which may be a potential limitation of this imaging modality.

\section{Power Doppler ultrasound}

MRI is the gold standard for quantitative deep soft-tissue imaging due to its unparalleled sensitivity, specificity, and clinical validation $[46,47]$. However, the prohibitively high costs of MRI and limited access have contributed to a marked increase in ultrasound (US) imaging. US has several advantages including real-time imaging, accessibility, cost-efficiency, and absence of ionizing radiation [48]. Moreover, US is particularly useful for assessment of RA joint inflammation with gray-scale imaging, and power Doppler (PD) US assessment of synovial hyperemia; both modalities assist in the diagnosis of active synovitis and flares while providing semiquantitative analyses (PD Score) of synovial inflammation for clinical research [49-52]. MRI cost-efficiency issues also apply to preclinical research [53-56], so we adapted PD-US imaging to murine models of inflammatory-erosive arthritis to assess synovitis and alterations of draining LNs longitudinally. Recently, we validated our PD-US 
quantitative assessments of expanding vs collapsed PLN in TNF-Tg mice [57, 58]. However, PD-US imaging of human LN, particularly PLN, remains elusive due to tissue depth concerns, and continues to be an area of active investigation.

\section{Near-infrared imaging of indocyanine green}

The availability of near-infrared (NIR) imaging of injected indocyanine green (ICG) fluorescent dye was a transformative advance in the quantitative assessment of in-vivo lymphatic function. Low-resolution NIR-ICG imaging has been used to identify sentinel LN during tumor resection surgery for a decade $[59,60]$, but appropriate optics with computer analyses to quantify human lymphatic function was introduced only recently. In these pioneering studies, groups such as that of SevickMuraca and colleagues applied NIR-ICG imaging techniques to evaluate lymphatic vessels in patients with lymphedema [61-63]. More recently, they examined 24 patients with lymphedema and 20 healthy controls, and demonstrated more tortuous and friable lymphatic vessels with extravascular accumulation of lymph in lymphedema patients compared with controls [64]. Furthermore, they quantified the contraction rate of the intrinsic contractile activity of lymphatic vessels in healthy patients. However, they were unable to calculate the contraction rate in lymphedema patients due to the dysmorphic vessel architecture and the inability to visualize ICG-labeled lymph movement in bolus formation, which is how the lymphatic contractions are quantified. Moreover, NIR-ICG imaging actually showed some retrograde movement of lymph in the lymphedema patients. This finding is likely due to the loss of normal vessel contractile ability followed by extravasation of lymph into the surrounding tissue and chronic tissue edema in these patients. A major question we plan to address is whether disturbances in RA lymphatic function are analogous to those observed in lymphedema patients.
The first publication describing NIR-ICG imaging in arthritis used infrared spectroscopy to study synovial fluid in patients with inflammatory arthritis versus osteoarthritis in order to differentiate the types of arthritis [65]. Preclinical studies using mouse models of arthritis reported that in-vivo arthritis detection in the early stages of disease activity is feasible with the use of NIR imaging and fluorescent-labeled molecules such as ICG and folate receptor-targeted dyes [66-68]. Moving towards clinical translation, Krohn et al. [69] examined the hands of 31 patients with untreated early RA using NIRICG optical imaging versus conventional MRI and US. Patients were given intravenous ICG and were assessed with a specialized imaging system and camera. They examined wrist, metacarpophalangeal (MCP), and proximal interphalangeal (PIP) joints and developed a 4-point scoring system to identify the degree of enhancement. The findings were correlated with MRI scoring of synovitis in the same joints. The results showed that NIR-ICG imaging correlates well with MRI findings at certain phases of ICG uptake, but it was inconsistent and not superior to current imaging modalities. However, the relative speed and simplicity of this minimally invasive procedure coupled with invivo real-time results demonstrated the potential of providing information to the clinician in a timely manner.

We are now performing a clinical pilot study of NIRICG imaging of the upper extremities in RA patients experiencing hand and/or wrist joint flares, and comparing the lymph flow data with that observed in healthy controls, to evaluate lymphatic vasculature and LNs in human subjects (ClinicalTrials.gov NCT02680067). In brief, a custom NIR imaging system is used to visualize lymphatic contractions after a negligible amount of ICG dye is injected into the web spaces of the hands. NIR excitation is monitored using a power meter. After the injections, the upper extremities are imaged continuously
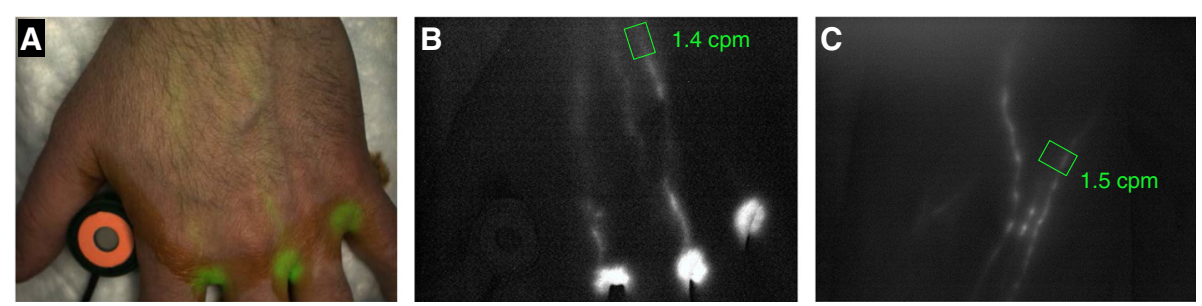

Fig. 3 Clinical NIR imaging to quantify lymphatic flow in the upper extremity. A custom NIR imaging system (FD-1665; FluxData Inc., Rochester, NY, USA) was used to assess lymphatic contraction frequency in a healthy human subject after ICG injection in the second, third, and fourth web spaces of both hands as described in ClinicalTrials.gov NCT2680067. NIR excitation $\left(<1.8 \mathrm{~mW} / \mathrm{cm}^{2}\right)$ was monitored with a Thorlabs PM16-121 power meter adjacent to the first web space. After injections, the upper extremities were imaged for 10 minutes to observe lymphatic flow. Visible and NIR (>800 nm) images were collected simultaneously; ICG fluorescence images were used to pseudocolor the visible image to provide anatomic localization (a). The ROI was positioned over lymphatics to calculate the mean pixel value for each NIR frame; peaks associated with lymphatic contractions were counted to calculate cpm. Representative images of the left hand (b) obtained from real-time video (Additional file 1: Movie 1) and right antecubital fossa (c) (Additional file 2: Movie 2) with the ROI and the respective cpm (green) 
for 10 minutes to observe lymphatic flow. A region of interest (ROI) is then positioned over lymphatics to calculate the mean pixel value; peaks associated with lymphatic contractions are indicative of contractions per minute (cpm). Figure 3 and Additional file 1: Movie 1 and Additional file 2: Movie 2 describe the technique and illustrate the sensitivity and specificity of the approach to quantify lymphatic contractions using this first-generation experimental approach.

The rationale for using NIR-ICG to evaluate lymphatic contractions is reasonable because it provides real-time information for the clinician. Furthermore, dysfunctional contractions, or no contractions, could indicate that the arthritic episode is the result of a drainage issue rather than synovial disturbances. The treatment would thus be tailored appropriately (i.e. with use of anti-TNF therapy), which has been associated with increased lymphatic angiogenesis [18] rather than DMARDs or steroids. Because of the extensive use of ICG in cardiovascular imaging over the decades, this dye has been studied extensively in humans and has been approved by the FDA for use as an imaging dye. There is therefore no major health limitation of the NIR-ICG technique. The only limitation is the duration of a study, which can take about 1-2 hours, the equivalent of current imaging techniques such as MRI.

\section{Conclusions}

The potential importance of lymphatic function as a key variable in RA flare is supported by the presence of palpable LN and lymphedema in some patients, and by preclinical data demonstrating major alterations in draining LNs and vasculature prior to arthritis onset. The role of the lymphatics in RA can now be examined with the advent of in-vivo imaging modalities that quantify lymphatic flow and contraction frequency. These technical advances may empower investigators to promote understanding in three critical areas. The first area is the redefinition of patterns of lymphatic flow anatomically, because most of our knowledge is derived from cadaveric studies and circulatory system mapping. Interestingly, our early studies have demonstrated inconsistencies in lymphatics drainage compared with previously published data. The second area is discovery of the cellular, molecular, and structural mechanisms that regulate lymphatic function and that are closely integrated with local biomechanics, inflammation, and parasympathetic innervation. Finally, the ultimate goal of this research is to identify novel molecular targets that will give rise to new interventions for RA flare. To achieve this goal, advancing technologies to noninvasively evaluate superficial and deep lymphatics in humans is a critical first step.

\section{Additional files}

Additional file 1: Video 1 of the left hand of a healthy patient after injection of ICG into the web spaces. The synchronized chart below is the mean fluorescent intensity (arbitrary units) within the green ROI. 20x real time. (M4V $2.64 \mathrm{mb}$ )

Additional file 2: Video 2 of the right arm of a healthy patient after injection of ICG into the web spaces. The synchronized chart below is the mean fluorescent intensity (arbitrary units) within the green ROI. 20x real time. (M4V $2.18 \mathrm{mb}$ )

\section{Acknowledgements}

HR is supported by grants from NIH (AR067885 and AR056702). RB and EMB are supported by a NIH training grant (AR053459). RWW is supported by a $\mathrm{NIH}$ grant (AR061307). LX is supported by NIH grants (AR048697, AR063650, and University of Rochester CTSA award UL1 TR000042), a grant from the National Natural Science Foundation of China (81220108027), and a grant from the Lymphatic Malformation Institute. EMS is supported by grants from $\mathrm{NIH}$ (P01 Al078907, AR056702 and AR061307).

\section{Competing interests}

$C T R$ receives consulting fees and research support from UCB

Pharmaceuticals. The remaining authors declare that they have no competing interests.

Authors' contributions

All authors read and approved the final manuscript.

\section{Ethics approval and consent to participate}

The studies cited in this publication (ClinicalTrials.gov NCT02680067. Registered 7 December 2015; ClinicalTrials.gov NCT01098201. Registered 30 March 2010; and ClinicalTrials.gov NCT01083563. Registered 8 March 2010) obtained ethics approval from the Research Subjects Review Board at the University of Rochester Medical Center. Informed, written consent to participate in the studies and consent for publication was obtained from all patients. All figures and video files in this publication are original for this article.

\section{Author details}

${ }^{1}$ Center for Musculoskeletal Research, University of Rochester School of Medicine and Dentistry, Rochester, NY, USA. ${ }^{2}$ Department of Pediatrics, University of Rochester School of Medicine and Dentistry, Rochester, NY, USA. ${ }^{3}$ Department of Pathology and Laboratory Medicine, University of Rochester School of Medicine and Dentistry, Rochester, NY, USA.

${ }^{4}$ Department of Biomedical Engineering, University of Rochester School of Medicine and Dentistry, Rochester, NY, USA. ${ }^{5}$ Department of Obstetrics and Gynecology, University of Rochester School of Medicine and Dentistry, Rochester, NY, USA. 'Department of Urology, University of Rochester School of Medicine and Dentistry, Rochester, NY, USA. 'Division of Allergy, Immunology, Rheumatology, Department of Medicine, University of Rochester School of Medicine and Dentistry, Rochester, NY, USA. ${ }^{8}$ Department of Neuroscience, University of Rochester School of Medicine and Dentistry, Rochester, NY, USA. ' University of Rochester Medical Center, 601 Elmwood Avenue, Box 777, Rochester, NY 14642, USA.

Published online: 01 September 2016

\section{References}

1. Gibofsky A. Epidemiology, pathophysiology, and diagnosis of rheumatoid arthritis: a synopsis. Am J Manag Care. 2014;20(7):S128-35.

2. Firestein GS. The disease formerly known as rheumatoid arthritis. Arthritis Res Ther. 2014;16(3):114.

3. Carubbi F, Zugaro L, Cipriani P, Conchiglia A, Gregori L, Danniballe C, Pistoia ML, Liakouli V, Ruscitti P, Ciccia F, et al. Safety and efficacy of intra-articular anti-tumor necrosis factor alpha agents compared to corticosteroids in a treat-to-target strategy in patients with inflammatory arthritis and monoarthritis flare. Int J Immunopathol Pharmacol. 2016;29(2):252-66.

4. Klasen IS, Ladestein RMT, Vandenberg WB, Benner R. Requirements for flare reactions of joint inflammation induced in mice by cloned Mt4+, Lyt-2- Tcells. Arthritis Rheum. 1989;32(3):330-7. 
5. Lens JW, Vandenberg WB, Vandeputte LBA, Zwarts WA. Flare of antigeninduced arthritis in mice after intravenous challenge - kinetics of antigen in the circulation and localization of antigen in the arthritic and noninflamed joint. Arthritis Rheum. 1986;29(5):665-74.

6. Bingham CO, Pohl C, Woodworth TG, Hewlett SE, May JE, Rahman MU, Witter JP, Furst DE, Strand CV, Boers M, et al. Developing a standardized definition for disease "flare" in rheumatoid arthritis (OMERACT 9 Special Interest Group). J Rheumatol. 2009;36(10):2335-41.

7. Benaglio F, Vitolo B, Scarabelli M, Binda E, Bugatti S, Caporali R, Montecucco C, Manzo A. The draining lymph node in rheumatoid arthritis: current concepts and research perspectives. Biomed Res Int. 2015;2015:420251.

8. Bouta EM, Li J, Ju Y, Brown EB, Ritchlin CT, Xing L, Schwarz EM. The role of the lymphatic system in inflammatory-erosive arthritis. Semin Cell Dev Biol. 2015;38:90-7.

9. Semo J, Nicenboim J, Yaniv K. Development of the lymphatic system: new questions and paradigms. Development. 2016;143(6):924-35.

10. Karpanen T, Alitalo K. Molecular biology and pathology of lymphangiogenesis. Annu Rev Pathol Mech. 2008;3:367-97.

11. Ionac M, Laskay T, Labahn D, Geisslinger G, Solbach W. Improved technique for cannulation of the murine thoracic duct: a valuable tool for the dissection of immune responses. J Immunol Methods. 1997;202(1):35-40.

12. Kaipainen A, Korhonen J, Mustonen T, Vanhinsbergh WWM, Fang GH, Dumont D, Breitman M, Alitalo K. Expression of the Fms-like tyrosine kinase4 gene becomes restricted to lymphatic endothelium during development. Proc Natl Acad Sci U S A. 1995;92(8):3566-70.

13. Joukov V, Pajusola K, Kaipainen A, Chilov D, Lahtinen I, Kukk E, Saksela O, Kalkkinen N, Alitalo K. A novel vascular endothelial growth factor, VEGF-C, is a ligand for the Flt4 (VEGFR-3) and KDR (VEGFR-2) receptor tyrosine kinases (vol 15, pg 290, 1996). Embo J. 1996;15(7):1751.

14. Zhou Q, Guo RL, Wood R, Boyce BF, Liang QQ, Wang YJ, Schwarz EM, Xing LP. Vascular endothelial growth factor $C$ attenuates joint damage in chronic inflammatory arthritis by accelerating local lymphatic drainage in mice. Arthritis Rheum. 2011;63(8):2318-28.

15. Zhang Q, Lu Y, Proulx ST, Guo RL, Yao ZQ, Schwarz EM, Boyce BF, Xing LP. Increased lymphangiogenesis in joints of mice with inflammatory arthritis. Arthritis Res Ther. 2007;9(6):R118.

16. Guo RL, Zhou Q, Proulx ST, Wood R, Ji RC, Ritchlin CT, Pytowski B, Zhu ZP, Wang YJ, Schwarz EM, et al. Inhibition of lymphangiogenesis and lymphatic drainage via vascular endothelial growth factor receptor 3 blockade increases the severity of inflammation in a mouse model of chronic inflammatory arthritis. Arthritis Rheum. 2009;60(9):2666-76.

17. Liang QQ, Ju YW, Chen Y, Wang WS, Li JL, Zhang L, Xu H, Wood RW, Schwarz EM, Boyce BF, et al. Lymphatic endothelial cells efferent to inflamed joints produce iNOS and inhibit lymphatic vessel contraction and drainage in TNF-induced arthritis in mice. Arthritis Res Ther. 2016;18:62.

18. Polzer K, Baeten D, Soleiman A, Distler J, Gerlag DM, Tak PP, Schett G, Zwerina J. Tumour necrosis factor blockade increases lymphangiogenesis in murine and human arthritic joints. Ann Rheum Dis. 2008;67(11):1610-6.

19. Young A, Koduri G. Extra-articular manifestations and complications of rheumatoid arthritis. Best Pract Res Clin Rheumatol. 2007;21(5):907-27.

20. Robertson MD, Hart FD, White WF, Nuki G, Boardman PL. Rheumatoid lymphadenopathy. Ann Rheum Dis. 1968;27(3):253-60.

21. Paulus HE, Machlede H, Peter JB, Goldberg L, Levy J, Pearson CM. Clinical improvement of rheumatoid-arthritis during prolonged thoracic-duct lymphocyte drainage. Arthritis Rheum. 1973;16(4):562-3.

22. Paulus HE, Machleder HI, Levine S, Yu DT, MacDonald NS. Lymphocyte involvement in rheumatoid arthritis. Studies during thoracic duct drainage. Arthritis Rheum. 1977;20(6):1249-62.

23. Paulus HE, Pearson CM, Peter JB, Stratton JA, Whitehou M, Yu D, Bangert $R$, Machlede $H$, Goldberg L. Effects of removing thoracic-duct lymphocytes from a patient with severe rheumatoid-arthritis. Arthritis Rheum. 1972;15(1): 122.

24. Paulus HE, Machlede H, Bangert R, Stratton JA, Goldberg L, Whitehou M, Yu $D$, Pearson CM. Case report-Thoracic-duct lymphocyte drainage in rheumatoid-arthritis. Clin Immunol Immunop. 1973;1 (2):173-81.

25. Pullinger BD, Florey HW. Proliferation of lymphatics in inflammation. J Path Bacteriol. 1937:45(1):157-70.

26. Andersson I, Marsal L, Nilsson B, Sjoblom KG, Wollheim FA. Abnormal axillary lymph nodes in rheumatoid arthritis. Acta Radiol Diagn (Stockh). 1980;21(5):645-9.

27. Olszewski WL, Pazdur J, Kubasiewicz E, Zaleska M, Cooke CJ, Miller NE. Lymph draining from foot joints in rheumatoid arthritis provides insight into local cytokine and chemokine production and transport to lymph nodes. Arthritis Rheum. 2001;44(3):541-9.

28. Kallioma J, Vastamak M. Chronic diffuse oedema of rheumatoid hand-a sign of local lymphatic involvement. Ann Rheum Dis. 1968;27(2):167.

29. Hart FD. Lymphadenopathy in rheumatic patients. Ann Rheum Dis. 1987; 46(8):645-6.

30. Bohm M, Riemann B, Luger TA, Bonsmann G. Bilateral upper limb lymphoedema associated with psoriatic arthritis: a case report and review of the literature. Br J Dermatol. 2000;143(6):1297-301.

31. Sant SM, Tormey VJ, Freyne P, Casey EB. Lymphatic obstruction in rheumatoid-arthritis. Clin Rheumatol. 1995;14(4):445-50.

32. Mulherin DM, Fitzgerald O, Bresnihan B. Lymphedema of the upper limb in patients with psoriatic-arthritis. Semin Arthritis Rheum. 1993;22(5):350-6.

33. Kiely PDW, Bland JM, Joseph AEA, Mortimer PS, Bourke BE. Upper-limb lymphatic function in inflammatory arthritis. J Rheumatol. 1995;22(2):214-7.

34. Proulx ST, Kwok E, You Z, Beck CA, Shealy DJ, Ritchlin CT, Boyce BF, Xing L, Schwarz EM. MRI and quantification of draining lymph node function in inflammatory arthritis. Ann N Y Acad Sci. 2007;1117:106-23.

35. Proulx ST, Kwok E, You Z, Papuga MO, Beck CA, Shealy DJ, Calvi LM, Ritchlin $\mathrm{CT}$, Awad HA, Boyce BF, et al. Elucidating bone marrow edema and myelopoiesis in murine arthritis using contrast-enhanced magnetic resonance imaging. Arthritis Rheum. 2008;58(7):2019-29.

36. Proulx ST, Kwok E, You Z, Papuga MO, Beck CA, Shealy DJ, Ritchlin CT, Awad $H A$, Boyce BF, Xing L, et al. Longitudinal assessment of synovial, lymph node, and bone volumes in inflammatory arthritis in mice by in vivo magnetic resonance imaging and microfocal computed tomography. Arthritis Rheum. 2007;56(12):4024-37.

37. McQueen FM. Magnetic resonance imaging in early inflammatory arthritis: what is its role? Rheumatology (Oxford). 2000;39(7):700-6.

38. Li J, Ju YW, Bouta EM, Xing LP, Wood RW, Kuzin I, Bottaro A, Ritchlin $\mathrm{CT}$, Schwarz EM. Efficacy of B cell depletion therapy for murine joint arthritis flare is associated with increased lymphatic flow. Arthritis Rheum. 2013;65(1):130-8.

39. Li J, Ritchlin CT, Sanz I, Bottaro A, Wood R, Xing LP, Schwarz EM. Effective anti-CD20 therapy for arthritis is associated with B-cell depletion in efferent lymph nodes and increased lymph and cellular flow in lymphatic vessels from arthritic joints. Arthritis Rheum. 2011;63(10):S975-6.

40. Zhou Q, Guo RL, Wood R, Proulx ST, Schwarz EM, Boyce BF, Xing LP. Blockade of lymphangiogenesis and lymphatic draining function enhances the severity of synovitis in mice with inflammatory-erosive arthritis. Arthritis Rheum. 2008;58(9):S504.

41. Li J, Kuzin I, Moshkani S, Proulx ST, Xing L, Skrombolas D, Dunn R, Sanz I, Schwarz EM, Bottaro A. Expanded CD23(+)/CD21(hi) B cells in inflamed lymph nodes are associated with the onset of inflammatory-erosive arthritis in TNF-transgenic mice and are targets of anti-CD20 therapy. J Immunol. 2010;184(11):6142-50.

42. Li J, Zhou Q, Wood R, Kuzin I, Bottaro A, Ritchlin C, Xing L, Schwarz E. CD23 $+/ C D 21$ hi B cell translocation and ipsilateral lymph node collapse is associated with asymmetric arthritic flare in TNF-Tg mice. Arthritis Res Ther. 2011;13(4):R138.

43. Bouta EM, Wood RW, Brown EB, Rahimi H, Ritchlin CT, Schwarz EM. In vivo quantification of lymph viscosity and pressure in lymphatic vessels and draining lymph nodes of arthritic joints in mice. J Physiol. 2014;592(Pt 6):1213-23.

44. Bremander $\mathrm{ABI}$, Petersson IF, Roos E. Validation of the rheumatoid arthritis outcome score (RAOS) - for the lower extremity. Health Qual Life Outcomes. 2003;1(1):1-11.

45. Huh YM, Kim S, Suh JS, Song HT, Song K, Shin KH. The role of popliteal lymph nodes in differentiating rheumatoid arthritis from osteoarthritis by using CE 3D-FSPGR MR imaging: relationship of the inflamed synovial volume. Korean J Radiol. 2005;6(2):117-24.

46. Gaffney K, Cookson J, Blades S, Coumbe A, Blake D. Quantitative assessment of the rheumatoid synovial microvascular bed by gadolinium-DTPA enhanced magnetic resonance imaging. Ann Rheum Dis. 1998;57(3):152-7.

47. Ostergaard M, Hansen M, Stoltenberg M, Gideon P, Klarlund M, Jensen KE, Lorenzen I. Magnetic resonance imaging-determined synovial membrane volume as a marker of disease activity and a predictor of progressive joint destruction in the wrists of patients with rheumatoid arthritis. Arthritis Rheum. 1999:42(5):918-29.

48. Tan YK, Ostergaard M, Conaghan PG. Imaging tools in rheumatoid arthritis: ultrasound vs magnetic resonance imaging. Rheumatology (Oxford). 2012; 51 Suppl 7:vii36-42. 
49. van de Stadt $L A$, Bos WH, Meursinge Reynders M, Wieringa $H$, Turkstra $F$, van der Laken CJ, van Schaardenburg D. The value of ultrasonography in predicting arthritis in auto-antibody positive arthralgia patients: a prospective cohort study. Arthritis Res Ther. 2010;12(3):R98.

50. Foltz V, Gandjbakhch F, Etchepare F, Rosenberg C, Tanguy ML, Rozenberg S, Bourgeois P, Fautrel B. Power Doppler ultrasound, but not low-field magnetic resonance imaging, predicts relapse and radiographic disease progression in rheumatoid arthritis patients with low levels of disease activity. Arthritis Rheum. 2012;64(1):67-76.

51. Peluso G, Michelutti A, Bosello S, Gremese E, Tolusso B, Ferraccioli G. Clinical and ultrasonographic remission determines different chances of relapse in early and long standing rheumatoid arthritis. Ann Rheum Dis. 2011;70(1):172-5.

52. Manzo A, Caporali R, Vitolo B, Alessi S, Benaglio F, Todoerti M, Bugatti S, Calliada F, Montecucco C. Subclinical remodelling of draining lymph node structure in early and established rheumatoid arthritis assessed by power Doppler ultrasonography. Rheumatology (Oxford). 2011;50(8):1395-400.

53. Clavel G, Marchiol-Fournigault C, Renault G, Boissier MC, Fradelizi D, Bessis N. Ultrasound and Doppler micro-imaging in a model of rheumatoid arthritis in mice. Ann Rheum Dis. 2008;67(12):1765-72.

54. Hammer HB, Sveinsson M, Kongtorp AK, Kvien TK. A 78-joints ultrasonographic assessment is associated with clinical assessments and is highly responsive to improvement in a longitudinal study of patients with rheumatoid arthritis starting adalimumab treatment. Ann Rheum Dis. 2010;69(7):1349-51.

55. Hartung W, Kellner H, Strunk J, Sattler H, Schmidt WA, Ehrenstein B, Fleck M, Backhaus M. Development and evaluation of a novel ultrasound score for large joints in rheumatoid arthritis: one year of experience in daily clinical practice. Arthritis Care Res (Hoboken). 2011;64(5):675-82.

56. Szkudlarek M, Court-Payen M, Jacobsen S, Klarlund M, Thomsen HS, Ostergaard M. Interobserver agreement in ultrasonography of the finger and toe joints in rheumatoid arthritis. Arthritis Rheum. 2003;48(4):955-62.

57. Ju Y, Rahimi H, Li J, Wood RW, Xing L, Schwarz EM. Validation of 3dimensional ultrasound versus magnetic resonance imaging quantification of popliteal lymph node volume as a biomarker of erosive inflammatory arthritis in mice. Arthritis Rheum. 2012;64(6):2048-50.

58. Bouta EM, Banik PD, Wood RW, Rahimi H, Ritchlin CT, Thiele RG, Schwarz EM. Validation of power Doppler versus contrast-enhanced magnetic resonance imaging quantification of joint inflammation in murine inflammatory arthritis. J Bone Miner Res. 2015;30(4):690-4.

59. Nimura H, Narimiya N, Mitsumori N, Yamazaki Y, Yanaga K, Urashima M. Infrared ray electronic endoscopy combined with indocyanine green injection for detection of sentinel nodes of patients with gastric cancer. Br J Surg. 2004;91(5):575-9.

60. Ito N, Fukuta M, Tokushima T, Nakai K, Ohgi S. Sentinel node navigation surgery using indocyanine green in patients with lung cancer. Surg Today. 2004;34(7):581-5.

61. Rasmussen JC, Tan IC, Marshall MV, Fife CE, Sevick-Muraca EM. Lymphatic imaging in humans with near-infrared fluorescence. Curr Opin Biotech. 2009;20(1):74-82.

62. Tan I, Maus E, Rasmussen J, Marshall M, Fife CE, Smith L, Sevick EM. Nearinfrared fluorescence imaging of lymphatics in head and neck lymphedema. J Nucl Med. 2011;52:101.

63. Tan IC, Maus EA, Rasmussen JC, Marshall MV, Adams KE, Fife CE, Smith LA, Chan W, Sevick-Muraca EM. Assessment of lymphatic contractile function after manual lymphatic drainage using near-infrared fluorescence imaging. Arch Phys Med Rehab. 2011;92(5):756-64.

64. Rasmussen JC, Tan IC, Marshall MV, Adams KE, Kwon S, Fife CE, Maus EA, Smith LA, Covington KR, Sevick-Muraca EM. Human lymphatic architecture and dynamic transport imaged using near-infrared fluorescence. Transl Oncol. 2010;3(6):362-72.

65. Shaw RA, Kotowich S, Eysel HH, Jackson M, Thomson GTD, Mantsch HH. Arthritis diagnosis based upon the near infrared spectrum of synovial fluid. Rheumatol Int. 1995;15(4):159-65.

66. Chen WT, Mahmood U, Weissleder R, Tung CH. Arthritis imaging using a nearinfrared fluorescence folate-targeted probe. Arthritis Res Ther. 2005;7(2):R310-7.

67. Hansch A, Frey O, Sauner D, Hilger I, Haas M, Malich A, Brauer R, Kaiser WA. In vivo imaging of experimental arthritis with near-infrared fluorescence. Arthritis Rheum. 2004;50(3):961-7.

68. Fischer T, Gemeinhardt I, Wagner S, von Stieglitz D, Schnorr J, Hermann KGA, Ebert B, Petzelt D, MacDonald R, Licha K, et al. Assessment of unspecific near-infrared dyes in laser-induced fluorescence imaging of experimental arthritis. Acad Radiol. 2006;13(1):4-13.
69. Krohn M, Ohrndorf S, Werner SG, Schicke B, Burmester GR, Hamm B, Backhaus M, Hermann KGA. Near-infrared fluorescence optical imaging in early rheumatoid arthritis: a comparison to magnetic resonance imaging and ultrasonography. J Rheumatol. 2015;42(7):1112-8. 